\title{
Natural rubber- carbon nanotubes composites, recent advances and challenges for electrical applications
}

\begin{abstract}
Over the last three decades, electrical and electronic industry is the leading industry in Malaysia in terms of export income and employment opportunities. However, to keep up with its international competition, Malaysia requires introducing novel improved products in this field. In this respects, using the natural resources available in the country is a fascinating option for developing electrical products. Natural rubber (NR), obtained from a tropical tree, has been widely considered as a base element in various electrical equipments. Despite of having excellent elasticity, original form of NR has low hardness, durability and tears resistance that limits its practical usage. Nanostructures, due their extraordinary characteristics, have indicated great improvements in NR-based composites. The results in terms of properties improvement are different depending on the types of nanofillers used. Among nanostructures, Carbon nanotubes (CNTs) have attracted a great attention in NR reinforcing. This paper presents the recent advances and state of the art for fabricating nanocomposites comprising NR and CNTs, as a sole filler or additive. The electrical properties of nanocomposite and its electrical applications are taken into account. The CNT/NR can be added to plastering materials for lightning protection of buildings, where high value of electrical conductivity is needed.
\end{abstract}

Keyword: CNT; Conductivity; Lightning protection; Nanocomposite; Natural rubber 